\title{
Black holes are one-dimensional
}

\author{
Jacob D. Bekenstein* and Avraham E. Mayo ${ }^{\dagger}$ \\ The Racah Institute of Physics, Hebrew University of Jerusalem, \\ Givat Ram, Jerusalem 91904, Israel
}

\begin{abstract}
The holographic principle has revealed that physical systems in 3-D space, black holes included, are basically two-dimensional as far as their information content is concerned. This conclusion is complemented by one sketched here: as far as entropy or information flow is concerned, a black hole behaves as a one-dimensional channel. We define a channel in flat spacetime in thermodynamic terms, and contrast it with common entropy emitting systems. A black hole is more like the former: its entropy output is related to the emitted power as it would be for a one-dimensional channel, and disposal of an information stream down a black hole is limited by the power invested in the same way as for a one-dimensional channel.
\end{abstract}

*e-mail: bekenste@vms.huji.ac.il

†e-mail:mayo@cc.huji.ac.il 
The holographic principle [1.2] claims that a generic physical system in three spatial dimensions is fundamentally two-dimensional. This idea is closely connected with the perception [2,3], now commonplace, that a black hole in 3-D space is really two-dimensional because its entropy qua measure of lost information is measured by the horizon's $2-\mathrm{D}$ area. In this essay we point out a further constriction of dimensions: viewed as an information absorber or entropy emitter, a black hole in 3-D is fundamentally one-dimensional, verily a portal to a one-dimensional information channel.

To show this one must define an information channel. In flat spacetime a channel is a complete set of unidirectionally propagating modes of a field parametrized by a single number. For example, all electromagnetic modes in free space with fixed wave vector direction and particular linear polarization constitute a channel, with the modes propagating in the specified sense parametrized solely by frequency. One might implement such a channel with a straight infinitely long coaxial cable (which is well known to transmit all frequencies) capped by polaroid filter at the entrance. Of course, one is not confined to electromagnetic field for sending information; sound (acoustic field) or neutrinos will do just as well. However, in this essay we mostly speak in terms of photons. A fundamental question is what is the maximum rate, in quantum theory, at which information may be transmitted in steady state down a photon channel for prescribed power $P$. The answer was found in the 1960's [4], but we reconstruct here the much later but very simple derivation of Pendry [5] because of its broad applicability.

One thinks of each possible signal state as represented by a particular occupation number state of the various propagating modes of the quantum electromagnetic field. Let us assume the channel is uniform in the direction of propagation; this allows us to label the modes by momentum $p$. One may allow for dispersion so that a quantum with momentum $p$ has some energy $\varepsilon(p)$. Then the propagation velocity of the quantum is the group velocity $v(p)=d \varepsilon(p) / d p$. According to information theory one can identify the information rate capacity for given $P$ with the maximal unidirectional thermodynamic entropy current that the channel can carry for that same $P$. This maximal entropy current obviously occurs for the thermal state, except that we must restrict attention to modes moving in a definite sense along the channel. We shall leave out the factor $\ln 2$ which translates from entropy natural units to bits.

Now the entropy $s(p)$ of any boson mode of momentum $p$ in a thermal state (temperature $T)$ is [6]

$$
s(p)=\frac{\varepsilon(p)}{e^{\varepsilon(p) / T}-1}-\ln \left(1-e^{-\varepsilon(p) / T}\right) .
$$

so the entropy current in one direction is

$$
\dot{S}=\int_{0}^{\infty} s(p) v(p) d p / 2 \pi \hbar,
$$

where $d p / 2 \pi \hbar$ is the number of modes per unit length in the interval $d p$ which propagate in one direction. This factor, when multiplied by the group velocity, gives the unidirectional current of modes. After an integration by parts on the second term coming from (2), we can cast the last result into the form

$$
\dot{S}=\frac{2}{T} \int_{0}^{\infty} \frac{\varepsilon(p)}{e^{\varepsilon(p) / T}-1} \frac{d \varepsilon(p)}{d p} \frac{d p}{2 \pi \hbar} .
$$


The first factor in the integrand is the mean energy per mode, so that the integral represents the unidirectional power $P$ in the channel. Thus

$$
\dot{S}=2 P / T
$$

The integral in Eq. (3) is evaluated by cancelling the two differentials $d p$ and assuming the energy spectrum is single valued and extends from 0 to $\infty$. Then the form of the dispersion relation $\varepsilon(p)$ does not enter and the result for the power is

$$
P=\pi(T)^{2} / 12 \hbar \text {. }
$$

Eliminating $T$ between the last two expressions gives Pendry's maximum entropy rate for power $P$,

$$
\dot{S}=(\pi P / 3 \hbar)^{1 / 2}
$$

The function $\dot{S}(P)$ in Eq. (6) is also called the noiseless quantum channel capacity. Surprisingly, it is independent, not only of the form of the mode velocity $v(p)$, but also of its scale. Thus the phonon channel capacity is as large as the photon channel capacity despite the difference in speeds. Why? Although phonons convey information at lower speed, the energy of a phonon is proportionately smaller than that of a photon in the equivalent mode. When the capacity is expressed in terms of the energy flux, or $P$, it turns out to involve the same constants. Formula. (6) neatly characterizes what we mean by one-dimensional transmission of entropy or information. It refers to transmission by use of a single species of quantum and a specific polarization; different species and polarizations engender separate channels.

For contrast let us derive, still in flat spacetime, the equivalent result for the energy and entropy transmission in a single photon polarization out of a closed hot black body surface with temperature $T$ and area $A$ into $3-D$ space. Halving the Stefan-Boltzmann law we have

$$
P=\frac{\pi^{2} T^{4} A}{120 h^{3}}
$$

as well as

$$
\dot{S}=4 P / 3 T
$$

whereby

$$
\dot{S}=\frac{2}{3}\left(\frac{2 \pi^{2} A P^{3}}{15 \hbar^{3}}\right)^{1 / 4}
$$

Our 3-D transmission system deviates from the sleek formula (6) not only in the exponent of $P$ but also in the appearance of the measure $A$ of the system. [In emission from a closed curve of length $L$ in two-dimensional space the factor $\left(L P^{2}\right)^{1 / 3}$ would appear instead of $\left.\left(A P^{3}\right)^{1 / 4}\right]$. In flat spacetime we may thus infer the dimensionality of the transmission system from the exponent of $P$ in the expression $\dot{S}(P)$ as well as from the value of the coefficient of $P / T$ in expressions for $\dot{S}$ like (4) or (8) $[(D+1) / D$ for $D$ space dimensions]. 
Turning now to curved spacetime, the radiation from a Schwarzschild black hole of mass $M$ in 3-D space is also given by Eqs. (7)-(8) with $A=4 \pi(2 M)^{2}$ and $T$ the Hawking temperature $T_{\mathrm{H}}=\hbar(8 \pi M)^{-1}$, except that we must augment the expression for $P$ by a factor $\bar{\Gamma}$ of order unity (Page [7] has calculated the frequency dependent barrier transmission factor $\Gamma$ and this must be averaged over the Planck spectrum to get $\bar{\Gamma}$ ), and replace the $4 / 3$ in the expression for $\dot{S}$ by another factor, $\nu$ (also calculated by Page [8]). Eliminating $M$ between the equations we have instead of Eq. (9)

$$
\dot{S}=\left(\frac{\nu^{2} \bar{\Gamma} \pi P}{480 \hbar}\right)^{1 / 2} .
$$

This looks completely different from the law (9) for the hot closed surface in 3-D space because, unlike for the hot body, a black hole's temperature is related to its mass in a specific way.

But (10) is of the same form as Pendry's limit (6) for one-channel flow. From Page's numerical estimates [7] we infer $\bar{\Gamma}=1.6267$ and take his value [8] $\nu=1.5003$, both for a single photon polarization. With these the numerical coefficient of (10) is $15.1 \%$ that of (6). One consequence of the above is that it is possible in principle to collect all the Hawking photon radiation (the former for one polarization) by means of suitable parabolic "mirrors" and "lenses" and pipe it down a single straight photon channel. This even though the black hole emits photons in all directions and thus, seemingly, into a gamut of channels. Evidently in its entropy emission properties, a black hole in 3-D space is more like a $1-\mathrm{D}$ channel than like a surface in 3-D space. We have checked that these conclusions are not qualitatively changed when the entropy is carried by neutrinos.

We have emphasized entropy flow out of the black hole; equally interesting is information flow into the black hole. One of the characteristics of a black hole is that it acts as a sink of information. Our results can be construed as putting a bound on how fast information may be disposed of into a black hole. Let us assume we have at our disposal a certain power $P$ to accomplish the task of getting rid of a stream of information. We may pick the size of black hole which suits us best. Then by the complementary relation between entropy and information, we may reinterpret formula (10) for entropy rate out of the black hole as also setting a bound on the rate at which information can flow into the black hole for given $P$ (there is actually a factor $\ln 2$ between them to convert entropy natural units to bits). The one-dimensional character of the black hole is central to this conclusion. One can, of course, improve the disposal rate by harnessing several channels (the second photon polarization, neutrinos, etc.), but the number of these is quite limited in nature. One must thus pay for information disposal into a black hole: the faster we want it done, the more power we have to put in, with the power growing quadratically with the loss of information rate.

Acknowledgments: This research was supported by grant No. 129/00-1 of the Israel Science Foundation. 


\section{REFERENCES}

[1] G. 't Hooft, in Salam-festschrifft, ed. A. Aly, J. Ellis and S. Randjbar-Daemi (World Scientific, Singapore 1993), gr-qc/9310026.

[2] L. Susskind, J. Math. Phys. 36, 6377 (1995).

[3] S. Corley and T. Jacobson, Phys. Rev. D53 6720 (1996).

[4] D. S. Lebedev and L. B. Levitin, Dokl. Akad. Nauk SSSR, 149, 1299 (1963) [translated in Sov. Phys. Dokl. 8, 377 (1963)].

[5] J. B. Pendry, Journ. Phys. A16, 2161 (1983).

[6] L. D. Landau and E. M. Lifshitz, Statistical Physics, Part I, third edition (Pergamon, Oxford 1980).

[7] D. N. Page, Phys. Rev. 13, 198 (1976).

[8] D. N. Page, Phys. Rev. Letters 50, 1013 (1983). 